\title{
On the Local Convergence of Regula-falsi-type Method for Generalized Equations
}

\author{
Farhana Alam ${ }^{*}$, M. H. Rashid ${ }^{2}$ and M. A. Alom ${ }^{3}$ \\ ${ }^{1}$ Department of Mathematics, University of Rajshahi, Rajshahi-6205, Bangladesh and Department of Computer \\ Science and Engineering, North Bengal International University, Rajshahi, Bangladesh \\ Email: chaiti_math@yahoo.com \\ ${ }^{2}$ Department of Mathematics, University of Rajshahi, Rajshahi-6205, Bangladesh \\ Email: harun_math@ru.ac.bd \\ ${ }^{3}$ Department of Mathematics, University of Rajshahi, Rajshahi-6205, Bangladesh and Department of \\ Mathematics, Khulna University of Engineering \& Technology, Khulna-9203, Bangladesh \\ Email: asraf .math.kuet@gmail.com
}

\begin{abstract}
Let $X$ and $Y$ be Banach spaces. We study a Regula-falsi-type method for solving the generalized equations $0 \in f(x)+g(x)+F(x)$, where $f: X \rightarrow Y$ is Fréchet differentiable in a neighborhood of a solution $\bar{x}, g: X \rightarrow Y$ is Fréchet differentiable at $\bar{x}$ and $F: X \rightrightarrows 2^{Y}$ is a set valued mapping with closed graph. Under some suitable assumptions, we prove the existence of any sequence generated by the Regula-falsi-type method and establish the local convergence results of the sequence generated by this method. Indeed, we will show that the sequence generated by this method converges linearly and super-linearly.
\end{abstract}

Keywords: Generalized equations, Set-valued mapping, Pseudo-Lipschitz continuity, Super-linear convergence, Divided difference, Local convergence.

\section{Introduction}

Let $X$ and $Y$ be Banach spaces. We deal with the problem of seeking a point $x \in X$ satisfying

$$
0 \in f(x)+g(x)+F(x),
$$

where $f: X \rightarrow Y$ is Fréchet differentiable in a neighborhood of a solution $\bar{x}$ of (1), $g: X \rightarrow Y$ is Fréchet differentiable at $\bar{x}$ but may be not differentiable in a neighborhood of $\bar{x}$ and $F: X \rightrightarrows 2^{Y}$ is a set valued mapping with closed graph.

It is clarified that when $F=\{0\},(1)$ is reduced to the classical problem of solving systems of nonlinear equations:

$$
f(x)+g(x)=0 .
$$

Cătinas [1] proposed the following method for solving (2) by using the combination of Newton's method with the secants method when $f$ is differentiable and $g$ is a continuous function admitting first and second order divided differences:

$$
0 \in f\left(x_{k}\right)+g\left(x_{k}\right)+\left(\nabla f\left(x_{k}\right)+\left[x_{k-1}, x_{k} ; g\right]\right)\left(x_{k+1}-x_{k}\right), \quad k=1,2, \ldots,
$$

where $\nabla f\left(x_{k}\right)$ denotes the Fréchet derivative of $f$ at $x$ and $[x, y ; g]$ the first order divided difference of $g$ on the points $x$ and $y$. To solve the problem (1), Geoffroy and Piétrus [2] extended the method (3) and proposed the following method:

$$
0 \in f\left(x_{k}\right)+g\left(x_{k}\right)+\left(\nabla f\left(x_{k}\right)+\left[x_{k-1}, x_{k} ; g\right]\right)\left(x_{k+1}-x_{k}\right)+F\left(x_{k+1}\right) .
$$

Under some assumptions, they proved that the sequence generated by this method converges superlinearly to the solution of (1). Next time for solving (1), Jean-Alexis and Piétrus [3] presented the following method:

$$
0 \in f\left(x_{k}\right)+g\left(x_{k}\right)+\left(\nabla f\left(x_{k}\right)+\left[2 x_{k+1}-x_{k}, x_{k} ; g\right]\right)\left(x_{k+1}-x_{k}\right)+F\left(x_{k+1}\right) .
$$


They proved that the sequence generated by (5) converges superlinearly by considering that $\nabla f$ and the first order divided difference of $g$ are $p$-Hölder continuous around a solution $\bar{x}$ and that $(f+g+F)^{-1}$ is pseudo-Lipschitz around $(0, \bar{x})$ with $F$ having closed graph. Rashid et al. [4] improved and extended the result, which has been given by Jean-Alexis and Piétrus [3] and show that if $\nabla f$ and the first order divided difference of $g$ are $p$-Hölder continuous at a solution $\bar{x}$, then the method (5) converges superlinearly.

It is remarkable that when $g=0,(1)$ is reduced to the following variational inclusion:

$$
0 \in f(x)+F(x) .
$$

Here (6) is a very general framework and many problems from applied mathematical areas, such as variational inequality problems including linear and nonlinear complementary problems, systems of nonlinear equations, abstract inequality systems and etc, can be cast as problem (6) (see $[5,6,7,8,10,11]$ ) and the references therein. In the case $g=0$, Geoffroy et al. [12] considered a second degree Taylor polynomial expansion of $f$ under suitable first and second order differentiability assumptions and showed that the existence of a sequence cubically converges to the solution of (1). When the single valued functions involved in (1) are differentiable, Newton-like methods can be considered to solve this problem, such an approach has been used in many contributions to this subject (see, e.g., $[4,8,11,13,14,15])$.

Let $x \in X$. The subset of $X$, denoted by $D(x)$, is defined by

$$
D_{x_{0}}(x)=\left\{d \in X: 0 \in f(x)+g(x)+\left(\nabla f(x)+\left[x_{0}, x ; g\right]\right) d+F(x+d)\right\} .
$$

To solve (1), Geoffroy and Piétrus [2, Theorem 3.1] considered two starting points $x_{0}$ and $x_{1}$ in a suitable neighborhood of $x^{*}$ and provided super-linear convergence result. In view of computational computations, this convergence is very slow because in their result, the terms $x_{k}$ and $x_{k-1}$ are involved in the upper bound of the relation $\left\|x_{k+1}-x^{*}\right\| \leq C\left\|x_{k}-x^{*}\right\| \max \left\{\left\|x_{k}-x^{*}\right\|,\left\|x_{k-1}-x^{*}\right\|\right\}$. This drawback motivates us to consider the following Regula-falsi-type method: Our aim is to prove the existence of a sequence

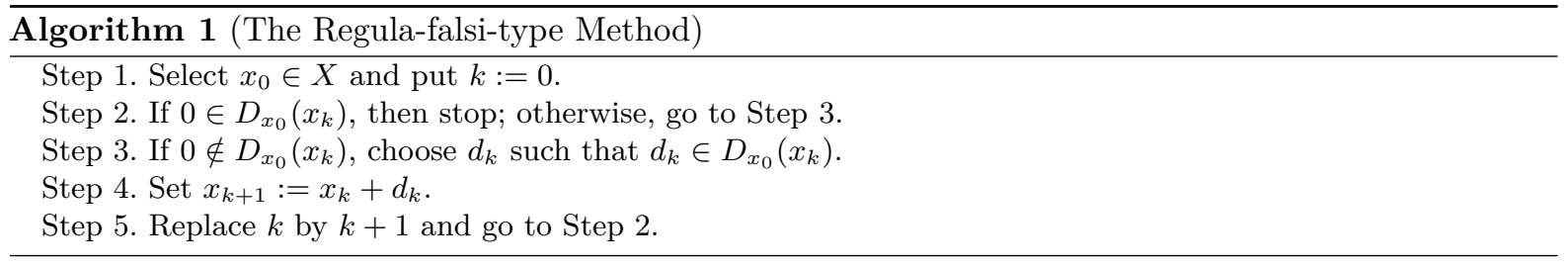

generated by Algorithm 1 which is locally linearly and superlinearly convergent to the solution $\bar{x}$ of (1). Geoffroy and Piétrus [2] considered two starting points $x_{0}$ and $x_{1}$ in a neighborhood of the solution to obtain their result, where as in our study we will consider one argument as a starting point $x_{0}$ in a neighborhood of the solution of (1).

The content of this paper is organized as follows: In section 2, we recall some necessary notations, notions and preliminary results. In section 3, we consider the Regula-falsi-type method to show the existence and convergence of the sequence generated by Algorithm 1. In the last section, we give a summary of the results obtained in this study.

\section{Notations and Preliminary Results}

Let $X$ and $Y$ be Banach spaces and let $F: X \rightrightarrows 2^{Y}$ be a set valued mapping. The graph of $F$ is defined by the $\operatorname{set} \operatorname{gph} F:=\{(x, y) \in X \times Y: y \in F(x)\}$ and the inverse of $F$ is defined by $F^{-1}(y):=\{x \in X: y \in F(x)\}$. Let $x \in X$ and $r>0$. By $\mathbb{B}(r, x)$, we denote the closed ball centered at $x$ with radius $r$, while $\mathcal{L}(X, Y)$ stands for the set of all bounded linear operators from $X$ to $Y$.

All the norms are denoted by $\|\cdot\|$. Let $B \subseteq X$ and $E \subseteq X$. The distance from a point $x$ to a set $B$ is defined by $\operatorname{dist}(x, B):=\inf \{\|x-a\|: a \in B\}$ for each $x \in X$, while the excess from a set $E$ to the set $B$ is defined by $e(E, B):=\sup \{\operatorname{dist}(x, B): x \in E\}$.

We take the definitions of the first and second divided difference operators from [2]: 
Definition 2.1. An operator belonging to the space $\mathcal{L}(X, Y)$ denoted by $\left[x_{0}, y_{0} ; g\right]$ is called the first order divided difference of the operator $g: X \rightarrow Y$ on the points $x_{0}, y_{0} \in X$ if both of the following properties hold:

(a) $\left[x_{0}, y_{0} ; g\right]\left(y_{0}-x_{0}\right)=g\left(y_{0}\right)-g\left(x_{0}\right)$ for $x_{0} \neq y_{0}$;

(b) If $g$ is Fréchet differentiable at $x_{0} \in X$ then $\left[x_{0}, x_{0} ; g\right]=g^{\prime}\left(x_{0}\right)$.

Definition 2.2. An operator belonging to the space $\mathcal{L}(X, \mathcal{L}(X, Y))$ denoted by $\left[x_{0}, y_{0}, z_{0} ; g\right]$ is called the second order divided difference of the operator $g: X \rightarrow Y$ on the points $x_{0}, y_{0}, z_{0} \in X$ if both of the following properties hold:

(a) $\left[x_{0}, y_{0}, z_{0} ; g\right]\left(z_{0}-x_{0}\right)=\left[y_{0}, z_{0} ; g\right]-\left[x_{0}, y_{0} ; g\right]$ for the distinct points $x_{0}, y_{0}$ and $z_{0}$;

(b) If $g$ is twice differentiable at $x_{0} \in X$ then $\left[x_{0}, x_{0}, x_{0} ; g\right]=\frac{g^{\prime \prime}\left(x_{0}\right)}{2}$.

We recall the definition of pseudo-Lipschitz continuity for set-valued mappings from [11] whose notion was introduced by Aubin [16] and has been studied extensively; see examples $[4,8,13,14]$ and the references therein.

Definition 2.3. Let $\Gamma: Y \rightrightarrows 2^{X}$ be a set-valued mapping and let $(\bar{y}, \bar{x}) \in \operatorname{gph} \Gamma$. Then $\Gamma$ is said to be pseudo-Lipschitz around $(\bar{y}, \bar{x})$ if there exist constants $r_{\bar{x}}>0, r_{\bar{y}}>0$ and $M>0$ such that the following inequality holds:

$$
e\left(\Gamma\left(y_{1}\right) \cap \mathbb{B}\left(r_{\bar{x}}, \bar{x}\right), \Gamma\left(y_{2}\right)\right) \leq M\left\|y_{1}-y_{2}\right\| \quad \text { for any } y_{1}, y_{2} \in \mathbb{B}\left(r_{\bar{y}}, \bar{y}\right) .
$$

We finish this section with the following lemma. This lemma is known as Banach fixed point lemma which has been proved by Dontchev and Hagger in [17]. This fixed-point lemma is the vital mechanism to prove the existence of any sequence generated by Algorithm 1.

Lemma 2.1. Let $\Phi: X \rightrightarrows 2^{X}$ be a set-valued mapping. Let $\eta_{0} \in X, r \in(0, \infty)$ and $\lambda \in(0,1)$ be such that

$$
\operatorname{dist}\left(\eta_{0}, \Phi\left(\eta_{0}\right)\right)<r(1-\lambda)
$$

and

$$
e\left(\Phi\left(x_{1}\right) \cap \mathbb{B}\left(r, \eta_{0}\right), \Phi\left(x_{2}\right)\right) \leq \lambda\left\|x_{1}-x_{2}\right\| \quad \text { for any } x_{1}, x_{2} \in \mathbb{B}\left(r, \eta_{0}\right) .
$$

Then $\Phi$ has a fixed point in $\mathbb{B}\left(r, \eta_{0}\right)$, that is, there exists $x \in \mathbb{B}\left(r, \eta_{0}\right)$ such that $x \in \Phi(x)$. If $\Phi$ is single-valued, then there exists $x \in \mathbb{B}\left(r, \eta_{0}\right)$ such that $x=\Phi(x)$.

\section{Convergence Analysis}

Suppose $X$ and $Y$ are Banach spaces. Let $\bar{x}$ be a solution of (1). This section is devoted to study the local convergence of the sequence generated by Algorithm 1. Let $r_{\bar{x}}>0$. We suppose that $F$ has closed graph, $f$ is Fréchet differentiable and its derivative is continuous in a neighborhood of $\bar{x}$ with a constant $\varepsilon>0$, $g$ is differentiable at $\bar{x}$ and admits a second order divided difference satisfying the following condition: there exists $\kappa>0$ such that for all $x, y, z \in \mathbb{B}\left(r_{\bar{x}}, \bar{x}\right),\|[x, y, z ; g]\| \leq \kappa$. To this end, let $x \in X$ and let us define the mapping $Q_{x}$ by

$$
Q_{x}(\cdot):=f(x)+\nabla f(x)(\cdot-x)+g(\cdot)+F(\cdot) .
$$

The following result establishes the equivalence relation between $(f+g+F)^{-1}$ and $Q_{\bar{x}}^{-1}$. This result is the refinement of the result in [9] or [8].

Lemma 3.1. Let $f: X \rightarrow Y$ be a function and let $(\bar{x}, \bar{y}) \in \operatorname{gph}(f+g+F)$. Assume that $f$ is Fréchet differentiable and its derivative is continuous in an open neighborhood $\Omega$ of $\bar{x}$ and $g$ admits a second order divided difference on $\Omega$. Then the followings are equivalent:

(i) The mapping $(f+g+F)^{-1}$ is pseudo-Lipschitz at $(\bar{y}, \bar{x})$;

(ii) The mapping $Q_{\bar{x}}^{-1}(\cdot)$ is pseudo-Lipschitz at $(\bar{y}, \bar{x})$. 
Proof. Define a function $h: X \rightarrow Y$ by

$$
h(x):=-f(x)+f(\bar{x})+\nabla f(\bar{x})(x-\bar{x}) .
$$

The proof is similar to that of [9], because the proof does not depend on the property of $g$.

Define a single valued mapping $Z_{x}: X \rightarrow Y$ by

$$
Z_{x}(y):=\left\{\begin{array}{c}
f(\bar{x})-f(x)+g(y)-g(x)+\nabla f(\bar{x})(y-\bar{x}) \\
-\left(\nabla f(x)+\left[x_{0}, x ; g\right]\right)(y-x), \text { for } y \neq x, \\
f(\bar{x})-f(x)+\nabla f(\bar{x})(y-\bar{x}), \text { for } y=x .
\end{array}\right.
$$

Define a set valued mapping $\Phi_{x}: X \rightrightarrows 2^{X}$ by

$$
\Phi_{x}(\cdot)=Q_{\bar{x}}{ }^{-1}\left[Z_{x}(\cdot)\right] .
$$

For every $x^{\prime}, x^{\prime \prime} \in X$, we have that

$$
\begin{aligned}
\left\|Z_{x}\left(x^{\prime}\right)-Z_{x}\left(x^{\prime \prime}\right)\right\| & =\left\|(\nabla f(\bar{x})-\nabla f(x))\left(x^{\prime}-x^{\prime \prime}\right)+g\left(x^{\prime}\right)-g\left(x^{\prime \prime}\right)-\left[x_{0}, x ; g\right]\left(x^{\prime}-x^{\prime \prime}\right)\right\| \\
& \leq\|\nabla f(\bar{x})-\nabla f(x)\|\left\|x^{\prime}-x^{\prime \prime}\right\|+\left\|\left[x^{\prime \prime}, x^{\prime} ; g\right]-\left[x_{0}, x ; g\right]\right\|\left\|x^{\prime}-x^{\prime \prime}\right\| \\
& =\left(\|\nabla f(\bar{x})-\nabla f(x)\|+\left\|\left[x^{\prime \prime}, x^{\prime} ; g\right]-\left[x_{0}, x ; g\right]\right\|\right)\left\|x^{\prime}-x^{\prime \prime}\right\| .
\end{aligned}
$$

\subsection{Linear Convergence}

The following lemma plays an important role to prove our first main theorem.

Lemma 3.2. Let $\bar{x}$ be a solution of (1). Suppose that $Q_{\bar{x}}^{-1}$ is pseudo-Lipschitz at $(0, \bar{x})$ with constant $M$. Assume that $\nabla f$ is continuous in the neighborhood of $\bar{x}$ with constant $\varepsilon>0$ and $g$ admits second order divided difference in the neighborhood of $\bar{x}$. Then, there exists $\delta>0$ such that for each $x \in \mathbb{B}(\delta, \bar{x})$, there is $\hat{x} \in \mathbb{B}(\delta, \bar{x})$ satisfying

$$
0 \in f(x)+g(x)+\left(\nabla f(x)+\left[x_{0}, x ; g\right]\right)(\hat{x}-x)+F(\hat{x})
$$

and

$$
\|\hat{x}-\bar{x}\| \leq \frac{1}{2}\|x-\bar{x}\|
$$

Proof. The $M$-pseudo-Lipschitz continuity of $Q_{\bar{x}}^{-1}$ around $(0, \bar{x})$ implies that there exist $r_{\bar{x}}>0$, and $r_{0}>0$ such that

$$
e\left(Q_{\bar{x}}^{-1}\left(y_{1}\right) \cap \mathbb{B}\left(r_{\bar{x}}, \bar{x}\right), Q_{\bar{x}}^{-1}\left(y_{2}\right)\right) \leq M\left\|y_{1}-y_{2}\right\| \text { for all } y_{1}, y_{2} \in \mathbb{B}\left(r_{0}, 0\right)
$$

Let $\varepsilon>0$ and $\kappa>0$ be such that

$$
M(\varepsilon+\kappa)<\frac{1}{6}
$$

The continuity property of $\nabla f$ and the second order divided difference of $g$ imply that there exists $\bar{r}>0$ such that

$$
\|\nabla f(y)-\nabla f(x)\| \leq \varepsilon \quad \text { for all } x, y \in \mathbb{B}(\bar{r}, \bar{x})
$$

and

$$
\|[x, y, z ; g]\| \leq \kappa \quad \text { for all } x, y, z \in \mathbb{B}(\bar{r}, \bar{x})
$$

Let $\delta>0$ be such that

$$
\delta \leq \min \left\{r_{\bar{x}}, \frac{r_{0}}{3 \varepsilon+4 \kappa}, \frac{2-3 M \varepsilon}{12 M \kappa}, 1\right\} .
$$

Fix $x \in X$ with $x \neq \bar{x}$, and define

$$
r_{x}:=3 M(\varepsilon+\kappa)\|x-\bar{x}\| .
$$


For $x \in \mathbb{B}(\delta, \bar{x})$ and using (16), we have

$$
r_{x} \leq 3 M(\varepsilon+\kappa) \delta<\delta
$$

We will apply Lemma 2.1 to the map $\Phi_{x}$ with $\eta_{0}:=\bar{x}$ and $r:=r_{x}$ and $\lambda:=\frac{2}{3}$ to conclude that there exists a fixed point $\hat{x} \in \mathbb{B}\left(r_{x}, \bar{x}\right)$ such that $\hat{x} \in \Phi_{x}(\hat{x})$, that is, $Z_{x}(\hat{x}) \in Q_{\bar{x}}(\hat{x})$, which implies that

$$
0 \in f(x)+g(x)+\left(\nabla f(x)+\left[x_{0}, x ; g\right]\right)(\hat{x}-x)+F(\hat{x}),
$$

i.e. (13) holds. Furthermore, $\hat{x} \in \mathbb{B}\left(r_{x}, \bar{x}\right) \subseteq \mathbb{B}(\delta, \bar{x})$ and so

$$
\|\hat{x}-\bar{x}\| \leq r_{x} \leq 3 M(\varepsilon+\kappa) \leq \frac{1}{2}\|x-\bar{x}\| .
$$

i.e. (14) holds. Thus, to complete the proof, it is sufficient to show that Lemma 2.1 is applicable for the map $\Phi_{x}$ with $\eta_{0}:=\bar{x}$ and $r:=r_{x}$ and $\lambda:=\frac{2}{3}$. To do this, it remains to prove that both assertions (8) and (9) of Lemma 2.1 hold. It is obvious that $\bar{x} \in Q_{\bar{x}}^{-1}(0) \cap \mathbb{B}\left(r_{x}, \bar{x}\right)$. According to the definition of the excess $e$, we have

$$
\begin{aligned}
\operatorname{dist}\left(\bar{x}, \Phi_{x}(\bar{x})\right) & \leq e\left(Q_{\bar{x}}^{-1}(0) \cap \mathbb{B}\left(r_{x}, \bar{x}\right), \Phi_{x}(\bar{x})\right) \\
& \leq e\left(Q_{\bar{x}}^{-1}(0) \cap \mathbb{B}(\delta, \bar{x}), Q_{\bar{x}}{ }^{-1}\left[Z_{x}(\bar{x})\right]\right) \\
& \leq e\left(Q_{\bar{x}}^{-1}(0) \cap \mathbb{B}\left(r_{\bar{x}, \bar{x}}\right), Q_{\bar{x}}^{-1}\left[Z_{x}(\bar{x})\right]\right) .
\end{aligned}
$$

For all $u \in \mathbb{B}\left(r_{x}, \bar{x}\right) \subseteq \mathbb{B}(\delta, \bar{x})$ with $u \neq x$, we have from (10) that

$$
\begin{aligned}
\left\|Z_{x}(u)\right\| & =\| f(\bar{x})-f(x)+g(u)-g(x)-\left(\nabla f(x)+\left[x_{0}, x ; g\right]\right)(u-x) \\
& +\nabla f(\bar{x})(u-\bar{x}) \| \\
& \leq\|f(\bar{x})-f(x)-\nabla f(\bar{x})(\bar{x}-x)\|+\|(\nabla f(\bar{x})-\nabla f(x))(u-x)\| \\
& +\left\|\left([x, u ; g]-\left[x_{0}, x ; g\right]\right)(u-x)\right\| .
\end{aligned}
$$

Since $f(\bar{x})-f(x)-\nabla f(\bar{x})(\bar{x}-x)=\int_{0}^{1}[\nabla f(\bar{x}+t(\bar{x}-x))-\nabla f(\bar{x})](\bar{x}-x) d t$, by using the continuity property of $\nabla f$ with constant $\varepsilon$, we have that

$$
\|f(\bar{x})-f(x)-\nabla f(\bar{x})(\bar{x}-x)\| \leq \varepsilon\|x-\bar{x}\| .
$$

Using (24) and the second order divided difference concept with a constant $\kappa$, we have from (23) that

$$
\begin{aligned}
\left\|Z_{x}(u)\right\| & \leq \varepsilon\|x-\bar{x}\|+\varepsilon\|u-x\|+\left\|\left[x_{0}, x, u ; g\right]\right\|\left\|u-x_{0}\right\|\|u-x\| \\
& \leq \varepsilon(\|x-\bar{x}\|+\|u-x\|)+\kappa\left\|u-x_{0}\right\|\|u-x\| \\
& \leq 3 \varepsilon \delta+4 \kappa \delta^{2}<3 \varepsilon \delta+4 \kappa \delta \\
& =(3 \varepsilon+4 \kappa) \delta<r_{0} .
\end{aligned}
$$

Therefore $Z_{x}(u) \in \mathbb{B}\left(r_{0}, 0\right)$ for all $u \in \mathbb{B}\left(r_{x}, \bar{x}\right)$ with $u \neq x$. Furthermore, in the case when $u=x$, we have from (23) that

$$
\left\|Z_{x}(x)\right\|=\|f(\bar{x})-f(x)-\nabla f(\bar{x})(x-\bar{x})\| .
$$

Using (24) and the third assumption from (19) in (26), we obtain

$$
\left\|Z_{x}(x)\right\| \leq \varepsilon\|x-\bar{x}\|=\varepsilon \delta<r_{0} .
$$

Thus we obtain

$$
Z_{x}(u) \in \mathbb{B}\left(r_{0}, 0\right) \quad \text { for all } u \in \mathbb{B}\left(r_{x}, \bar{x}\right)
$$


On the other hand, letting $u=\bar{x}$ in (23) and for $\delta \leq 1$ by (19), we obtain

$$
\begin{aligned}
\left\|Z_{x}(\bar{x})\right\| & \leq\|f(\bar{x})-f(x)-\nabla f(x)(x-\bar{x})\|+\left\|\left([x, \bar{x} ; g]-\left[x_{0}, x ; g\right]\right)(\bar{x}-x)\right\| \\
& \left.\leq\|f(\bar{x})-f(x)-\nabla f(x)(x-\bar{x})\|+\left\|[x, \bar{x} ; g]-\left[x_{0}, x ; g\right]\right\| \| \bar{x}-x\right) \| \\
& \leq \varepsilon\|x-\bar{x}\|+\left\|\left[x_{0}, x, \bar{x} ; g\right]\right\|\left\|\bar{x}-x_{0}\right\|\|\bar{x}-x\| \\
& \leq\left(\varepsilon+\kappa\left\|x_{0}-\bar{x}\right\|\right)\|x-\bar{x}\| \leq(\varepsilon+\kappa \delta)\|x-\bar{x}\| \\
& \leq(\varepsilon+\kappa)\|x-\bar{x}\| .
\end{aligned}
$$

This together with (15) and (22) (with $y_{1}=0$ and $\left.y_{2}=Z_{x}(\bar{x})\right)$ implies that

$$
\begin{aligned}
\operatorname{dist}\left(\bar{x}, \Phi_{x}(\bar{x})\right) & \leq M\left\|Z_{x}(\bar{x})\right\| \\
& \leq M(\varepsilon+\kappa)\|x-\bar{x}\| \\
& =\left(1-\frac{2}{3}\right) r_{x}=(1-\lambda) r .
\end{aligned}
$$

This shows that the assertion (8) of Lemma 2.1 is satisfied.

Now, we show that assertion (9) of Lemma 2.1 is also satisfied. Let $x^{\prime}, x^{\prime \prime} \in \mathbb{B}\left(r_{x}, \bar{x}\right)$. Then by (21), we have that $x^{\prime}, x^{\prime \prime} \in \mathbb{B}\left(r_{x}, \bar{x}\right) \subseteq \mathbb{B}(\delta, \bar{x})$ and hence $Z_{x}\left(x^{\prime}\right), Z_{x}\left(x^{\prime \prime}\right) \in \mathbb{B}\left(r_{0}, 0\right)$ by $(27)$. This together with (15) (with $y_{1}=Z_{x}\left(x^{\prime}\right)$ and $y_{2}=Z_{x}\left(x^{\prime \prime}\right)$ ) implies that

$$
\begin{aligned}
e\left(\Phi_{x}\left(x^{\prime}\right)\right. & \left.\cap \mathbb{B}\left(r_{x}, \bar{x}\right), \Phi_{x}\left(x^{\prime \prime}\right)\right) \leq e\left(\Phi_{x}\left(x^{\prime}\right) \cap \mathbb{B}(\delta, \bar{x}), \Phi_{x}\left(x^{\prime \prime}\right)\right) \\
& \leq e\left(Q_{\bar{x}}^{-1}\left[Z_{x}\left(x^{\prime}\right)\right] \cap \mathbb{B}\left(r_{\bar{x}}, \bar{x}\right), Q_{\bar{x}}^{-1}\left[Z_{x}\left(x^{\prime \prime}\right)\right]\right) \\
& \leq M\left\|Z_{x}\left(x^{\prime}\right)-Z_{x}\left(x^{\prime \prime}\right)\right\| .
\end{aligned}
$$

In the case $x^{\prime} \neq x^{\prime \prime}$, since $g$ admits second order divided difference, (18) is applicable to concluding that

$$
\begin{aligned}
& \left(\left\|\left[x^{\prime \prime}, x^{\prime} ; g\right]-\left[x_{0}, x ; g\right]\right\|\right)\left\|x^{\prime}-x^{\prime \prime}\right\| \\
= & \left(\left\|\left[x^{\prime \prime}, x^{\prime} ; g\right]-\left[x, x^{\prime \prime} ; g\right]+\left[x, x^{\prime \prime} ; g\right]-\left[x_{0}, x ; g\right]\right\|\right)\left\|x^{\prime}-x^{\prime \prime}\right\| \\
= & \left(\left\|\left[x, x^{\prime \prime}, x^{\prime} ; g\right]\left(x^{\prime}-x\right)+\left[x_{0}, x, x^{\prime \prime} ; g\right]\left(x^{\prime \prime}-x_{0}\right)\right\|\right)\left\|x^{\prime}-x^{\prime \prime}\right\| \\
\leq & \left(\left\|\left[x, x^{\prime \prime}, x^{\prime} ; g\right]\right\|\left\|x^{\prime}-x\right\|+\left\|\left[x_{0}, x, x^{\prime \prime} ; g\right]\right\|\left\|x^{\prime \prime}-x_{0}\right\|\right)\left\|x^{\prime}-x^{\prime \prime}\right\| \\
\leq & 4 \kappa \delta\left\|x^{\prime}-x^{\prime \prime}\right\| .
\end{aligned}
$$

This, together with (12) and (17), gives that

$$
\begin{aligned}
\left\|Z_{x}\left(x^{\prime}\right)-Z_{x}\left(x^{\prime \prime}\right)\right\| & \leq\left(\|\nabla f(\bar{x})-\nabla f(x)\|+\left\|\left[x^{\prime \prime}, x^{\prime} ; g\right]-\left[x_{0}, x ; g\right]\right\|\right)\left\|x^{\prime}-x^{\prime \prime}\right\| \\
& \leq(\varepsilon+4 \delta \kappa)\left\|x^{\prime}-x^{\prime \prime}\right\| .
\end{aligned}
$$

Combining this with (28) and using the relation $12 M \kappa \delta \leq 2-3 M \varepsilon$ by (19), we obtain that

$$
\begin{aligned}
e\left(\Phi_{x}\left(x^{\prime}\right)\right. & \left.\cap \mathbb{B}\left(r_{x}, \bar{x}\right), \Phi_{x}\left(x^{\prime \prime}\right)\right) \\
& \leq M(\varepsilon+4 \delta \kappa)\left\|x^{\prime}-x^{\prime \prime}\right\| \\
& \leq \frac{2}{3}\left\|x^{\prime}-x^{\prime \prime}\right\|=\lambda\left\|x^{\prime}-x^{\prime \prime}\right\| .
\end{aligned}
$$

This implies that assertion (9) of Lemma 2.1 is satisfied. This completes the proof of the Lemma.

The following theorem shows that the sequence $\left\{x_{n}\right\}$ generated by Algorithm 1 converges linearly. 
Theorem 3.1. Let $\bar{x}$ be a solution of (1). Suppose that $Q_{\bar{x}}^{-1}$ is pseudo-Lipschitz at $(0, \bar{x})$ with constant $M$. Assume that $\nabla f$ is continuous in the neighborhood of $\bar{x}$ with constant $\varepsilon>0$ and $g$ admits second order divided difference in the neighborhood of $\bar{x}$. Then, there exists $\delta>0$ such that for every starting point $x_{0} \in \mathbb{B}(\delta, \bar{x})$, there is a sequence $\left\{x_{n}\right\}$ generated by Algorithm 1 converges to $\bar{x}$ and which satisfies that

$$
\left\|x_{k+1}-\bar{x}\right\| \leq \frac{1}{2}\left\|x_{k}-\bar{x}\right\| \quad \text { for each } k=0,1,2, \ldots
$$

Proof. By Lemma 3.2, there exists $\delta>0$ such that

$$
\text { for each } x \in \mathbb{B}(\delta, \bar{x}) \Longrightarrow \text { there is } \hat{x} \in \mathbb{B}(\delta, \bar{x}) \text { such that (13) and (14) hold. }
$$

Let $x_{0} \in \mathbb{B}(\delta, \bar{x})$. Then, it follows from (30) that there exists $\hat{x} \in \mathbb{B}(\delta, \bar{x})$ such that $\hat{x} \in \Phi_{x_{0}}(\hat{x})$. This implies that $Z_{x_{0}}(\hat{x}) \in Q_{\bar{x}}(\hat{x})$, which translates to

$$
0 \in f\left(x_{0}\right)+g\left(x_{0}\right)+\left(\nabla f\left(x_{0}\right)+\left[x_{0}, x_{0} ; g\right]\right)\left(\hat{x}-x_{0}\right)+F(\hat{x})
$$

and

$$
\|\hat{x}-\bar{x}\| \leq \frac{1}{2}\left\|x_{0}-\bar{x}\right\|
$$

Thus, (31) indicates that $D_{x_{0}}\left(x_{0}\right) \neq \emptyset$. Consequently, we can choose $d_{0} \in D_{x_{0}}\left(x_{0}\right)$. By Algorithm 1 , $x_{1}:=x_{0}+d_{0}$ is defined. Thus, we obtain from (31) and (32) that

$$
0 \in f\left(x_{0}\right)+g\left(x_{0}\right)+\left(\nabla f\left(x_{0}\right)+\left[x_{0}, x_{0} ; g\right]\right)\left(x_{1}-x_{0}\right)+F\left(x_{1}\right)
$$

and

$$
\left\|x_{1}-\bar{x}\right\| \leq \frac{1}{2}\left\|x_{0}-\bar{x}\right\|
$$

and so (29) holds for $k=0$. We will proceed by induction on $k$. Now we assume that $x_{0}, x_{1}, \ldots, x_{k}$ are generated by Algorithm 1 satisfying (29). Then by (30), we have that $D_{x_{k}}\left(x_{k}\right) \neq \emptyset$. Then by Algorithm 1 , we can select $d_{k} \in D_{x_{0}}\left(x_{k}\right)$ such that $x_{k+1}=x_{k}+d_{k}$. Then (31) and (32) give that

$$
0 \in f\left(x_{k}\right)+g\left(x_{k}\right)+\left(\nabla f\left(x_{k}\right)+\left[x_{0}, x_{k} ; g\right]\right)\left(x_{k+1}-x_{k}\right)+F\left(x_{k+1}\right)
$$

and

$$
\left\|x_{k+1}-\bar{x}\right\| \leq \frac{1}{2}\left\|x_{k}-\bar{x}\right\|
$$

and so (29) holds for all $k$. This completes the proof of the Theorem.

\subsection{Superlinear Convergence}

This section is devoted to study the superlinear convergence of the sequence generated by Algorithm 1 . Let $r>0$ and we assume that $\nabla f$ is Lipschitz continuous in a neighborhood $\mathbb{B}(r, \bar{x})$ of the solution $\bar{x}$ of (1), that is, there exists $L>0$ such that

$$
\|\nabla f(y)-\nabla f(x)\| \leq L\|x-y\|, \quad \text { for all } x, y \in \mathbb{B}(\bar{r}, \bar{x})
$$

and $g$ admits second order divided difference, that is, there exists $\kappa>0$ such that

$$
\|[x, y, z ; g]\| \leq \kappa \quad \text { for all } x, y, z \in \mathbb{B}(\bar{r}, \bar{x}) .
$$

We assume that $L$ and $\kappa$ are related in such a way that

$$
L<\frac{1-4 M \kappa}{7 M} \text {. }
$$

Set

$$
\gamma:=\frac{7 M(L+4 \kappa)}{4}
$$

It follows from (35) that $\gamma<\frac{1}{4}<1$.

The following lemma is an analogue of Lemma 3.2. However, the proof technique is slightly different. 
Lemma 3.3. Let $\bar{x}$ be a solution of (1). Assume that $Q_{\bar{x}}^{-1}$ is pseudo-Lipschitz at $(0, \bar{x})$ with constant M. Suppose that $\nabla f$ is Lipschitz continuous in the neighborhood of $\bar{x}$ with constant $L>0$ and $g$ admits second order divided difference in the neighborhood of $\bar{x}$ with constant $\kappa>0$. Let $\gamma$ be defined by (36). Then, there exists $\delta>0$ such that for each $x \in \mathbb{B}(\delta, \bar{x})$, there is $\hat{x} \in \mathbb{B}(\delta, \bar{x})$ satisfying

$$
0 \in f(x)+g(x)+\left(\nabla f(x)+\left[x_{0}, x ; g\right]\right)(\hat{x}-x)+F(\hat{x})
$$

and

$$
\|\hat{x}-\bar{x}\| \leq \gamma\|x-\bar{x}\| \max \left\{\|x-\bar{x}\|,\left\|x_{0}-\bar{x}\right\|\right\} .
$$

Proof. By the assumed pseudo-Lipschitz property of $Q_{\bar{x}}^{-1}$ with constant $M>0$ around $(0, \bar{x})$ implies that there exist $r_{\bar{x}}>0$, and $r_{0}>0$ such that

$$
e\left(Q_{\bar{x}}^{-1}\left(y_{1}\right) \cap \mathbb{B}\left(r_{\bar{x}}, \bar{x}\right), Q_{\bar{x}}^{-1}\left(y_{2}\right)\right) \leq M\left\|y_{1}-y_{2}\right\| \text { for all } y_{1}, y_{2} \in \mathbb{B}\left(r_{0}, 0\right) .
$$

Let $\delta>0$ be such that

$$
\delta \leq \min \left\{r_{\bar{x}},\left(\frac{2 r_{0}}{5 L+8 \kappa}\right)^{\frac{1}{2}}, \frac{1}{7 M(L+4 \kappa)}, 1\right\} .
$$

Fix $x \in X$ with $x \neq \bar{x}$, and define

$$
r_{x}:=\gamma\|x-\bar{x}\| \max \left\{\|x-\bar{x}\|,\left\|x_{0}-\bar{x}\right\|\right\} .
$$

It follows for $x \in \mathbb{B}(\delta, \bar{x})$ that

$$
r_{x} \leq \frac{7 M(L+4 \kappa)}{4} \delta \max \{\delta, \delta\}=\frac{7 M(L+4 \kappa) \delta^{2}}{4}
$$

This, together with the relation $7 M(L+4 \kappa) \delta \leq 1$ in $(40)$, implies that

$$
r_{x} \leq \frac{7 M(L+4 \kappa) \delta}{4} \delta \leq \delta
$$

The proof will be completed if we can apply Lemma 2.1 to the map $\Phi_{x}$ with $\eta_{0}:=\bar{x}$ and $r:=r_{x}$ and $\lambda:=\frac{1}{7}$ to show that there exists a fixed point $\hat{x} \in \mathbb{B}\left(r_{x}, \bar{x}\right)$ such that $\hat{x} \in \Phi_{x}(\hat{x})$, that is, $Z_{x}(\hat{x}) \in Q_{\bar{x}}(\hat{x})$. This implies that

$$
0 \in f(x)+g(x)+\left(\nabla f(x)+\left[x_{0}, x ; g\right]\right)(\hat{x}-x)+F(\hat{x}),
$$

i.e. $D_{x_{0}}(x) \neq \emptyset$ and hence (37) holds. Furthermore, $\hat{x} \in \mathbb{B}\left(r_{x}, \bar{x}\right) \subseteq \mathbb{B}(\delta, \bar{x})$ and so

$$
\|\hat{x}-\bar{x}\| \leq r_{x}=\gamma\|x-\bar{x}\| \max \left\{\|x-\bar{x}\|,\left\|x_{0}-\bar{x}\right\|\right\}
$$

i.e. (38) holds. Thus, to finish the proof, it is sufficient to show that Lemma 2.1 is applicable for the map $\Phi_{x}$ with $\eta_{0}:=\bar{x}$ and $r:=r_{x}$ and $\lambda:=\frac{1}{7}$. To do this, it remains to prove that both assertions $(8)$ and $(9)$ of Lemma 2.1 hold. It is obvious that $\bar{x} \in Q_{\bar{x}}^{-1}(0) \cap \mathbb{B}\left(r_{x}, \bar{x}\right)$. According to the definition of the excess $e$, we have

$$
\begin{aligned}
\operatorname{dist}\left(\bar{x}, \Phi_{x}(\bar{x})\right) & \leq e\left(Q_{\bar{x}}^{-1}(0) \cap \mathbb{B}\left(r_{x}, \bar{x}\right), \Phi_{x}(\bar{x})\right) \\
& \leq e\left(Q_{\bar{x}}^{-1}(0) \cap \mathbb{B}(\delta, \bar{x}), Q_{\bar{x}}{ }^{-1}\left[Z_{x}(\bar{x})\right]\right) \\
& \leq e\left(Q_{\bar{x}}^{-1}(0) \cap \mathbb{B}\left(r_{\bar{x}, \bar{x}}\right), Q_{\bar{x}}^{-1}\left[Z_{x}(\bar{x})\right]\right) .
\end{aligned}
$$


For all $u \in \mathbb{B}\left(r_{x}, \bar{x}\right) \subseteq \mathbb{B}(\delta, \bar{x})$ with $u \neq x$, we have from (10) that

$$
\begin{aligned}
\left\|Z_{x}(u)\right\| & =\| f(\bar{x})-f(x)+g(u)-g(x)-\left(\nabla f(x)+\left[x_{0}, x ; g\right]\right)(u-x) \\
& +\nabla f(\bar{x})(u-\bar{x}) \| \\
& \leq\|f(\bar{x})-f(x)-\nabla f(\bar{x})(\bar{x}-x)\|+\|(\nabla f(\bar{x})-\nabla f(x))(u-x)\| \\
& +\left\|\left([x, u ; g]-\left[x_{0}, x ; g\right]\right)(u-x)\right\| \\
& \leq\|f(\bar{x})-f(x)-\nabla f(\bar{x})(\bar{x}-x)\|+\|\nabla f(\bar{x})-\nabla f(x)\|\|u-x\| \\
& +\left\|[x, u ; g]-\left[x_{0}, x ; g\right]\right\|\|u-x\| .
\end{aligned}
$$

Note by (33) that

$$
\begin{aligned}
& \|f(\bar{x})-f(x)-\nabla f(\bar{x})(\bar{x}-x)\| \\
= & \left\|\int_{0}^{1}[\nabla f(\bar{x}+t(\bar{x}-x))-\nabla f(\bar{x})](\bar{x}-x) d t\right\| \\
\leq & \int_{0}^{1}\|\nabla f(\bar{x}+t(\bar{x}-x))-\nabla f(\bar{x})\|\|\bar{x}-x\| d t \\
\leq & L \int_{0}^{1} t\|\bar{x}-x\|^{2} d t=\frac{L}{2}\|\bar{x}-x\|^{2} .
\end{aligned}
$$

This together with the relation $(5 L+8 \kappa) \delta^{2} \leq 2 r_{0}$ by $(40)$ and notion of second order divided difference with a constant $\kappa$, we have from (44) that

$$
\begin{aligned}
\left\|Z_{x}(u)\right\| & \leq \frac{L}{2}\|x-\bar{x}\|^{2}+L\|\bar{x}-x\|\|u-x\|+\left\|\left[x_{0}, x, u ; g\right]\right\|\left\|u-x_{0}\right\|\|u-x\| \\
& \leq\left(\frac{L}{2}+2 L+4 \kappa\right) \delta^{2}=\frac{5 L+8 \kappa}{2} \delta^{2} \\
& <r_{0} .
\end{aligned}
$$

It follows that for all $u \in \mathbb{B}\left(r_{x}, \bar{x}\right)$ with $u \neq x, Z_{x}(u) \in \mathbb{B}\left(r_{0}, 0\right)$. Furthermore, in the case when $u=x$, we have from (44) that

$$
\left\|Z_{x}(x)\right\|=\|f(\bar{x})-f(x)-\nabla f(\bar{x})(x-\bar{x})\| .
$$

Using (45) and the third relation from (40) in (47), we obtain

$$
\left\|Z_{x}(x)\right\| \leq \frac{L}{2}\|x-\bar{x}\|^{2}=\frac{L \delta^{2}}{2}<r_{0} .
$$

This implies that $Z_{x}(u) \in \mathbb{B}\left(r_{0}, 0\right)$, for all $u \in \mathbb{B}\left(r_{x}, \bar{x}\right)$.

On the other hand, letting $u=\bar{x}$ in (44) and for $\delta \leq 1$ by (40), we obtain

$$
\begin{aligned}
\left\|Z_{x}(\bar{x})\right\| & \leq\|f(\bar{x})-f(x)-\nabla f(\bar{x})(\bar{x}-x)\|+\|\nabla f(\bar{x})-\nabla f(x)\|\|\bar{x}-x\| \\
& +\left\|[x, \bar{x} ; g]-\left[x_{0}, x ; g\right]\right\|\|\bar{x}-x\| \\
& \leq \frac{L}{2}\|x-\bar{x}\|^{2}+L\|\bar{x}-x\|^{2}+\left\|\left[x_{0}, x, \bar{x} ; g\right]\right\|\left\|\bar{x}-x_{0}\right\|\|\bar{x}-x\| \\
& \leq \frac{3 L}{2}\|x-\bar{x}\|^{2}+\kappa\left\|x_{0}-\bar{x}\right\|\|x-\bar{x}\| \\
& \leq \frac{(3 L+2 \kappa)}{2}\|x-\bar{x}\| \max \left\{\|x-\bar{x}\|,\left\|x_{0}-\bar{x}\right\|\right\} \\
& <\frac{3(L+4 \kappa)}{2}\|x-\bar{x}\| \max \left\{\|x-\bar{x}\|,\left\|x_{0}-\bar{x}\right\|\right\} .
\end{aligned}
$$

This together with (39) and (43) (with $y_{1}=0$ and $y_{2}=Z_{x}(\bar{x})$ ) implies that

$$
\begin{aligned}
\operatorname{dist}\left(\bar{x}, \Phi_{x}(\bar{x})\right) & \leq M\left\|Z_{x}(\bar{x})\right\| \\
& \leq \frac{3 M(L+4 \kappa)}{2}\|x-\bar{x}\| \max \left\{\|\| x-\bar{x}\left\|, \mid x_{0}-\bar{x}\right\|\right\} \\
& =\left(1-\frac{1}{7}\right) r_{x}=(1-\lambda) r .
\end{aligned}
$$


This shows that the assertion (8) of Lemma 2.1 is satisfied.

Now, we verify that assertion (9) of Lemma 2.1 is also satisfied. Let $x^{\prime}, x^{\prime \prime} \in \mathbb{B}\left(r_{x}, \bar{x}\right)$. Then by (42), we have that $x^{\prime}, x^{\prime \prime} \in \mathbb{B}\left(r_{x}, \bar{x}\right) \subseteq \mathbb{B}(\delta, \bar{x})$ and hence $Z_{x}\left(x^{\prime}\right), Z_{x}\left(x^{\prime \prime}\right) \in \mathbb{B}\left(r_{0}, 0\right)$ by (46). This together with (39) (with $y_{1}=Z_{x}\left(x^{\prime}\right)$ and $y_{2}=Z_{x}\left(x^{\prime \prime}\right)$ ) implies that

$$
\begin{aligned}
e\left(\Phi_{x}\left(x^{\prime}\right)\right. & \left.\cap \mathbb{B}\left(r_{x}, \bar{x}\right), \Phi_{x}\left(x^{\prime \prime}\right)\right) \leq e\left(\Phi_{x}\left(x^{\prime}\right) \cap \mathbb{B}(\delta, \bar{x}), \Phi_{x}\left(x^{\prime \prime}\right)\right) \\
& \leq e\left(Q_{\bar{x}}^{-1}\left[Z_{x}\left(x^{\prime}\right)\right] \cap \mathbb{B}\left(r_{\bar{x}}, \bar{x}\right), Q_{\bar{x}}^{-1}\left[Z_{x}\left(x^{\prime \prime}\right)\right]\right) \\
& \leq M\left\|Z_{x}\left(x^{\prime}\right)-Z_{x}\left(x^{\prime \prime}\right)\right\| .
\end{aligned}
$$

In the case $x^{\prime} \neq x^{\prime \prime}$, since $g$ admits second order divided difference, (34) is applicable to concluding that

$$
\begin{aligned}
& \left(\left\|\left[x^{\prime \prime}, x^{\prime} ; g\right]-\left[x_{0}, x ; g\right]\right\|\right)\left\|x^{\prime}-x^{\prime \prime}\right\| \\
= & \left(\left\|\left[x^{\prime \prime}, x^{\prime} ; g\right]-\left[x, x^{\prime \prime} ; g\right]+\left[x, x^{\prime \prime} ; g\right]-\left[x_{0}, x ; g\right]\right\|\right)\left\|x^{\prime}-x^{\prime \prime}\right\| \\
= & \left(\left\|\left[x, x^{\prime \prime}, x^{\prime} ; g\right]\left(x^{\prime}-x\right)+\left[x_{0}, x, x^{\prime \prime} ; g\right]\left(x^{\prime \prime}-x_{0}\right)\right\|\right)\left\|x^{\prime}-x^{\prime \prime}\right\| \\
\leq & \left(\left\|\left[x, x^{\prime \prime}, x^{\prime} ; g\right]\right\|\left\|x^{\prime}-x\right\|+\left\|\left[x_{0}, x, x^{\prime \prime} ; g\right]\right\|\left\|x^{\prime \prime}-x_{0}\right\|\right)\left\|x^{\prime}-x^{\prime \prime}\right\| \\
\leq & 4 \kappa \delta\left\|x^{\prime}-x^{\prime \prime}\right\| .
\end{aligned}
$$

This, together with (12) and (33), gives that

$$
\begin{aligned}
\left\|Z_{x}\left(x^{\prime}\right)-Z_{x}\left(x^{\prime \prime}\right)\right\| & \leq\left(\|\nabla f(\bar{x})-\nabla f(x)\|+\left\|\left[x^{\prime \prime}, x^{\prime} ; g\right]-\left[x_{0}, x ; g\right]\right\|\right)\left\|x^{\prime}-x^{\prime \prime}\right\| \\
& \leq(L\|\bar{x}-x\|+4 \kappa \delta)\left\|x^{\prime}-x^{\prime \prime}\right\| \\
& \leq(L+4 \kappa) \delta\left\|x^{\prime}-x^{\prime \prime}\right\| .
\end{aligned}
$$

Combining this with (48) and using the relation $7 M(L+4 \kappa) \delta \leq 1$ by $(40)$, we obtain that

$$
\begin{aligned}
e\left(\Phi_{x}\left(x^{\prime}\right)\right. & \left.\cap \mathbb{B}\left(r_{x}, \bar{x}\right), \Phi_{x}\left(x^{\prime \prime}\right)\right) \\
& \leq M(L+4 \kappa) \delta\left\|x^{\prime}-x^{\prime \prime}\right\| \\
& \leq \frac{1}{7}\left\|x^{\prime}-x^{\prime \prime}\right\|=\lambda\left\|x^{\prime}-x^{\prime \prime}\right\| .
\end{aligned}
$$

This implies that assertion (9) of Lemma 2.1 is satisfied. This completes the proof of the Lemma.

Theorem 3.2. Let $\bar{x}$ be a solution of (1). Assume that $Q_{\bar{x}}^{-1}$ is pseudo-Lipschitz at $(0, \bar{x})$ with constant M. Suppose that $\nabla f$ is Lipschitz continuous in the neighborhood of $\bar{x}$ with constant $L>0$ and $g$ admits second order divided difference in the neighborhood of $\bar{x}$. Let $\gamma$ be defined by (36). Then, there exists $\delta>0$ such that for every starting point $x_{0} \in \mathbb{B}(\delta, \bar{x})$, there is a sequence $\left\{x_{n}\right\}$ generated by Algorithm 1 converges to $\bar{x}$ and which satisfies that

$$
\left\|x_{k+1}-\bar{x}\right\| \leq \gamma\left\|x_{k}-\bar{x}\right\| \max \left\{\left\|x_{k}-\bar{x}\right\|,\left\|x_{0}-\bar{x}\right\|\right\} \text { for each } k=0,1,2, \ldots
$$

Proof. By Lemma 3.3, there exists $\delta>0$ such that

$$
\text { for each } x \in \mathbb{B}(\delta, \bar{x}) \Longrightarrow \text { there is } \hat{x} \in \mathbb{B}(\delta, \bar{x}) \text { such that (37) and (38) hold. }
$$

Let $x_{0} \in \mathbb{B}(\delta, \bar{x})$. Then, it follows from (50) that there exists $\hat{x} \in \mathbb{B}(\delta, \bar{x})$ such that $\hat{x} \in \Phi_{x_{0}}(\hat{x})$. This implies that $Z_{x_{0}}(\hat{x}) \in Q_{\bar{x}}(\hat{x})$, which translates to

$$
0 \in f\left(x_{0}\right)+g\left(x_{0}\right)+\left(\nabla f\left(x_{0}\right)+\left[x_{0}, x_{0} ; g\right]\right)\left(\hat{x}-x_{0}\right)+F(\hat{x})
$$

and

$$
\|\hat{x}-\bar{x}\| \leq \gamma\left\|x_{0}-\bar{x}\right\| \max \left\{\left\|x_{0}-\bar{x}\right\|,\left\|x_{0}-\bar{x}\right\|\right\} .
$$


Thus, (51) indicates that $D_{x_{0}}\left(x_{0}\right) \neq \emptyset$. Consequently, we can choose $d_{0} \in D_{x_{0}}\left(x_{0}\right)$. By Algorithm 1 , $x_{1}:=x_{0}+d_{0}$ is defined. Thus, we obtain from (51) and (52) that

$$
0 \in f\left(x_{0}\right)+g\left(x_{0}\right)+\left(\nabla f\left(x_{0}\right)+\left[x_{0}, x_{0} ; g\right]\right)\left(x_{1}-x_{0}\right)+F\left(x_{1}\right)
$$

and

$$
\left\|x_{1}-\bar{x}\right\| \leq \gamma\left\|x_{0}-\bar{x}\right\| \max \left\{\left\|x_{0}-\bar{x}\right\|,\left\|x_{0}-\bar{x}\right\|\right\} .
$$

and so (49) holds for $k=0$. We will proceed by induction on $k$. Now we assume that $x_{0}, x_{1}, \ldots, x_{k}$ are generated by Algorithm 1 satisfying (49). Then by (50), we have that $D_{x_{k}}\left(x_{k}\right) \neq \emptyset$. Then by Algorithm 1 , we can select $d_{k} \in D_{x_{0}}\left(x_{k}\right)$ such that $x_{k+1}=x_{k}+d_{k}$. Then (51) and (52) give that

$$
0 \in f\left(x_{k}\right)+g\left(x_{k}\right)+\left(\nabla f\left(x_{k}\right)+\left[x_{0}, x_{k} ; g\right]\right)\left(x_{k+1}-x_{k}\right)+F\left(x_{k+1}\right)
$$

and

$$
\left\|x_{k+1}-\bar{x}\right\| \leq \gamma\left\|x_{k}-\bar{x}\right\| \max \left\{\left\|x_{k}-\bar{x}\right\|,\left\|x_{0}-\bar{x}\right\|\right\} .
$$

and so (49) holds for all $k$. This completes the proof of the Theorem.

\section{Concluding Remarks}

We have established local convergence results for Regula-falsi-type method for solving the generalized equation (1) under some suitable assumptions. When $Q_{\bar{x}}^{-1}$ is pseudo-Lipschitz continuous, $\nabla f$ is continuous and, $g$ admits second order divided difference, we have shown that the sequence generated by Algorithm 1 converges linearly. However, when $Q_{\bar{x}}^{-1}$ is pseudo-Lipschitz continuous, $\nabla f$ is Lipschitz continuous and, $g$ admits second order divided difference, we have presented super-linear convergence results of the Regula-falsi-type method defined by Algorithm 1. This study extends and improves the results corresponding to [2].

\section{Acknowledgments}

This work is supported financially by the Ministry of Education, Bangladesh, whose grant number is 37.20.0000.004.033.013.2015.

\section{References}

1. E. Cătinas, On some iterative methods for solving nonlinear equations, Rev. Anal. Numér. Théor. Approx., 23 (1994), 17-53.

2. M.H. Geoffroy, A. Piétrus, Local convergence of some iterative methods for solving generalized equations, J. Math. Anal. Appl., 290, (2004), 497-505.

3. C. Jean-Alexis, A. Pietrus, On the convergence of some methods for variational inclusions, Rev. R. Acad. Cien. serie A. Mat., 102(2), (2008), 355-361.

4. M.H. Rashid, J.H. Wang and C. Li, Convergence Analysis of a method for variational inclusions, Applicable Analysis, 91(10), (2012), 1943-1956.

5. A.L. Dontchev and R.T. Rockafellar, Regularity and conditioning of solution mappings in variational analysis, Set-valued Anal., 12(1), (2004), 79-109.

6. M.C. Ferris and J.S. Pang, Engineering and economic applications of complimentarity problems, SIAM Rev., 39, (1997), 669-713.

7. J.S. He, J.H. Wang and C. Li, Newton's method for undetermined systems of equations under the modified $\gamma$-condition, Numer. Funct. Anal. Optim., 28, (2007), 663-679.

8. M.H. Rashid, On the convergence of extended Newton-type method for solving variational inclusions, Journal of Cogent Mathematics, 1(1), (2014), 1-19.

9. M.H. Rashid, A. Sardar, Convergence of the Newton-type method for generalized equations, GANIT J. Bangladesh Math. Soc., 35 (2015), 27-40.

10. M.H. Rashid, Convergence analysis of gauss-type proximal point method for variational inequalities, Open Science Journal of Mathematics and Application, 2(1), (2014), 5-14. 
11. M.H. Rashid, S.H. Yu, C. Li, \& S.Y. Wu, Convergence analysis of the Gauss-Newton-type method for Lipschitz-like mappings, J. Optim. Theory Appl., 158(1), (2013), 216-233.

12. M.H. Geoffroy, S. Hilout and A. Pietrus, Acceleration of convergence in Dontchev's iterative methods for solving variational inclusions, Serdica Math. J., 2003, 45-54.

13. A.L. Dontchev, Local convergence of the Newton method for generalized equation, C. R. A. S Paris Ser.I, 322, (1996), 327-331.

14. A.L. Dontchev, Uniform convergence of the Newton method for Aubin continuous maps, Serdica Math. J., 22, (1996), 385-398.

15. C. Li and K.F. Ng, Majorizing functions and convergence of the Gauss-Newton method for convex composite optimization, SIAM J. Optim., 18, (2007), 613-642.

16. J.P. Aubin and H. Frankowska, Set-valued analysis, Birkhäuser, Boston, 1990.

17. A.L. Dontchev and W.W. Hager, An inverse mapping theorem for set-valued maps, Proc. Amer. Math. Soc., $121,(1994), 481-498$

18. M. Shub, S. Smale, Complexity of Bezout's Theorem 1: geometric aspects, J. Amer. Math. Soc., 6(1993), 459-501.

19. J. C. Yakoubsohn, Finding zeros of analytic functions: $\alpha$-theory for secant type method, J. Complexity, 15 (1999), 239-281. 